\title{
Diagnostic challenges in chikungunya infection: Report of an atypical presentation
}

\author{
Craig $\mathrm{J}^{1}$, Klowak $\mathrm{M}^{2}$, Boggild $\mathrm{AK}^{1,3,4^{\star}}$ \\ ${ }^{1}$ Division of Infectious Diseases, Department of Medicine, University of Toronto, Toronto, ON \\ ${ }^{2}$ Faculty of Life Sciences, McMaster University, Toronto, ON \\ ${ }^{3}$ Tropical Disease Unit, University Health Network-Toronto General Hospital, Toronto, ON \\ ${ }^{4}$ Public Health Ontario Laboratories, Public Health Ontario, Toronto, ON \\ *Corresponding author: andrea.boggild@utoronto.ca
}

\begin{abstract}
Due in part to increasing global travel, chikungunya fever has emerged as a significant public health concern. With recent outbreaks in Caribbean nations and the first report of locally acquired infection in the United States, there is concern that we may see an increasing number of cases in Canada. As chikungunya fever shares many clinical similarities to other arthropod-borne illnesses such as dengue fever, clinical diagnosis is challenging. We report an atypical presentation of acute chikungunya fever in a man returning from travel to Haiti. Microbiologic diagnosis, treatment, prognosis, and public health implications will aid clinician preparedness for this emerging pathogen.
\end{abstract}

\section{Introduction}

Chikungunya is a mosquito-borne viral disease increasingly recognized globally as an emerging pathogen. In December 2013, local transmission of chikungunya virus was reported for the first time in the Americas, leading the Public Health Agency of Canada (PHAC) to alert clinicians and public heath personnel to the possibility of chikungunya in returning travellers presenting with fever and polyarthralgia (1). A recent report from the United States Centers for Disease Control and Prevention (CDC) has identified 25 Caribbean countries with reported cases of autochthonous chikungunya infection (2). In this article, we report an atypical case of chikungunya infection in a traveller returning from Haiti, to highlight clinical features shared by chikungunya and other arthropod-borne infections such as dengue, the important diagnostic tools available to clinicians, and to address concerns around global spread of chikungunya infection.

\section{Background}

Chikungunya infection is caused by an alphavirus of the Togaviridae family, and is transmitted primarily by Aedes aegypti mosquitoes, and to a lesser extent, Aedes albopictus (3).

Chikungunya virus was first described in Tanzania in 1953 (4) and is considered endemic to parts of West Africa. As of September 2014, there were 88 countries reporting transmission of chikungunya infection, including those in Africa, Europe, Oceania, Asia and, most recently, the Americas (5). The first autochthonous infection with chikungunya in a temperate region occurred in Italy in 2007 with a suspected index case originating in India (6). The suspected vector, Aedes albopictus, was thought to have acquired a genetic mutation due to ecological pressure allowing it to supplement Aedes aegypti as a primary vector (3). As Aedes albopictus is widespread in the southeastern United States, there is growing concern about local transmission in these states. In fact, the first case of locally acquired chikungunya infection in the United States was recently reported in a man from Florida (7). Transmission is not expected to occur in Canada, as the Aedes mosquitoes are not found in this climate region (1).

In most cases, the presenting symptom is a disabling symmetrical polyarthralgia associated with fever (8). Common joints involved include the ankles, knees, metacarpophalangeal joints, metatarsal joints, shoulders, elbows, and wrists. In approximately one third of patients, joints can be swollen, although effusive arthritis is rare 
(8). Following a period of one to three days, there is often development of a diffuse maculopapular rash, usually sparing the face. Arthralgias typically resolve over weeks; however, in many cases, they can persist for months or even years, often having a significant impact on patient quality of life (9).

Clinical diagnosis is challenging as the signs and symptoms of chikungunya overlap with other illnesses, such as parvovirus B19 infection and dengue fever. Microbiologic confirmation is required, and is usually made through detection of immunoglobulin M (IgM) or immunoglobulin G ( $\lg G)$ antibodies in serum via enzyme-linked immunosorbent assay (ELISA). IgM antibodies are often detected two to six days after onset of symptoms, whereas IgG antibodies usually appear during the convalescent stage of illness and can persist for years (10). Reverse transcription polymerase chain reaction (RT-PCR), performed on serum, plasma or cerebral spinal fluid (CSF), offers the greatest sensitivity and is available through investigational use by the National Microbiology Laboratory in Winnipeg, Manitoba (11). Our case will highlight some of the diagnostic uncertainty surrounding the diagnosis of chikungunya.

Treatment for chikungunya fever is generally supportive, consisting of non-steroidal anti-inflammatory agents, fluids and rest. Corticosteroids are reserved for debilitating arthritis early in the course of acute infection (3). Research into potential monoclonal antibody treatment, antiviral therapy, and vaccinations is ongoing $(12,13)$.

\section{The case}

A 74-year-old man presented to the emergency department with constipation, abdominal pain, and a new onset diffuse non-desquamating maculopapular rash over the chest, back, arms and legs, following an 11-day trip to Haiti from which he returned one day prior to presentation. His rash was non-painful and non-pruritic. A computed tomography (CT) scan was performed of the abdomen showing small bowel diverticular inflammation and possible perforation into the surrounding fatty tissue. He was admitted to hospital for supportive care, including administration of antibiotics. His perforation was presumed secondary to a previous diagnosis of small bowel diverticular disease complicated by significant constipation.

While in Haiti, he had worked as an aid worker in a local health clinic. Prior to travel, he had completed vaccination series for both hepatitis $A$ and $B$ and had been prescribed antimalarial prophylaxis with chloroquine, to which he had been adherent. On day nine of his travel, he awoke with severe diffuse arthralgia affecting both large and small joints in the upper and lower extremities, rigors, and subjective fever. He had no respiratory or gastrointestinal complaints. His arthralgia dramatically improved over a 48-hour period, following which he developed a truncal rash as well as significant constipation, necessitating his presentation to the emergency department.

On physical examination in the emergency department, the patient's abdomen was soft and non-tender. There were no joint swellings noted; however, a maculopapular rash was seen over the chest, and the upper and lower extremities. Cardiac, respiratory and neurologic exams were normal. Routine laboratory investigations were performed (Table 1). A significant lymphopenia and thrombocytopenia were noted; chest radiography was also performed on admission and was normal.

Table 1: Routine laboratory investigations upon admission to hospital ${ }^{1}$

\begin{tabular}{|c|c|c|}
\hline Investigation & Value & Reference range \\
\hline Hemoglobin & $143 \mathrm{~g} / \mathrm{L}$ & $132-170 \mathrm{~g} / \mathrm{L}$ \\
\hline Leukocytes & $9.9 \times 10^{9} / L$ & $3.5-10 \times 10^{9} / \mathrm{L}$ \\
\hline - Neutrophils & $8.6 \times 10^{9} / \mathrm{L}$ & $2.5-7.5 \times 10^{9} / \mathrm{L}$ \\
\hline - Lymphocytes & $0.5 \times 10^{9} / L$ & $1.0-4.0 \times 10^{9} / \mathrm{L}$ \\
\hline Platelets & $108 \times 10^{9} / L$ & $130-400 \times 10^{9} / \mathrm{L}$ \\
\hline AST & $36 \mathrm{U} / \mathrm{L}$ & $13-37 \mathrm{U} / \mathrm{L}$ \\
\hline$\overline{A L T}$ & $18 \mathrm{U} / \mathrm{L}$ & $10-40 \mathrm{U} / \mathrm{L}$ \\
\hline ALP & $94 \mathrm{U} / \mathrm{L}$ & $40-120 \mathrm{U} / \mathrm{L}$ \\
\hline Bilirubin (total) & $11 \mathrm{mmol} / \mathrm{L}$ & $3.0-20 \mathrm{mmol} / \mathrm{L}$ \\
\hline Sodium & $136 \mathrm{mmol} / \mathrm{L}$ & $135-145 \mathrm{mmol} / \mathrm{L}$ \\
\hline Potassium & $4 \mathrm{mmol} / \mathrm{L}$ & $3.5-5.0 \mathrm{mmol} / \mathrm{L}$ \\
\hline Bicarbonate & $22 \mathrm{mmol} / \mathrm{L}$ & $20-30 \mathrm{mmol} / \mathrm{L}$ \\
\hline Creatinine & $79 \mathrm{mmol} / \mathrm{L}$ & $55-105 \mathrm{mmol} / \mathrm{L}$ \\
\hline
\end{tabular}




\begin{tabular}{|l|l|l|}
\hline Lactate & $1.0 \mathrm{mmol} / \mathrm{L}$ & $0.5-2.0 \mathrm{mmol} / \mathrm{L}$ \\
\hline Urine Cultures & Negative & $\mathrm{N} / \mathrm{A}^{2}$ \\
\hline Blood Cultures & Negative & $\mathrm{N}^{2}$ \\
\hline Malaria Rapid Antigen Test & $\mathrm{A}^{2}$ & $\mathrm{~N} / \mathrm{A}^{2}$ \\
\hline
\end{tabular}

${ }^{\mathrm{T}}$ Abnormal values are in bold

${ }^{2} \mathrm{~N} / \mathrm{A}=$ Not applicable

${ }^{3}$ Note: Microscopy of Giemsa-stained thin blood smears was also performed.

The patient received supportive care, including intravenous crystalloids, and completed a course of antibiotics in hospital. He was discharged with urgent referral to a tropical diseases clinic for evaluation of his presumed travelrelated illness. Dengue virus IgG and IgM antibodies were negative by ELISA. Stool cultures were negative for Salmonella spp., Escherichia coli 0157:H7, Campylobacter spp., and Shigella spp. Chikungunya IgM antibody was positive by ELISA supporting a probable diagnosis of acute chikungunya infection. Without follow-up confirmatory testing (such as by plaque reduction neutralization test), the possibility of cross-reactivity with other alphaviruses could not be definitively excluded. His abdominal pain resolved in hospital with supportive care alone, while his arthritis and rash completely resolved over a two-week period.

\section{Discussion}

We present an atypical case of acute infection with chikungunya in a man returning from Haiti, an area of known ongoing intense dengue and chikungunya transmission. Although the patient's initial symptoms included classic findings of symmetric polyarthritis and subsequent maculopapular rash, his clinical course was complicated by significant abdominal pain, constipation, as well as thrombocytopenia-features atypical of chikungunya. His dramatic improvement over 48 hours is also unusual as polyarthralgia leading to mobility and dexterity issues will frequently last for weeks to months (3). Although uncommon, other atypical manifestations of chikungunya infection have been reported in the literature. These include neurological features (including encephalitis, seizures, and Guillain-Barre syndrome), cardiovascular features (including myocarditis, heart failure, and ischemic heart disease), renal features (including acute kidney injury), ocular features (including optic neuritis), as well as atypical skin eruptions, ulcerations, and bullae (14).

The differential diagnosis of fever and non-effusive polyarthritis is broad. Common bacterial causes include Lyme disease and infective endocarditis. Frequent viral causes include parvovirus B19, hepatitis B and C, rubella, dengue and other alphaviruses, including Mayaro, O'nyong-nyong, Ross River, Barmah Forest, Sindbis, and Semliki Forest virus. Non-infectious etiologies include seronegative spondyloarthropathies, rheumatoid arthritis, crystal-induced arthropathies, and post-infectious (reactive) arthritis. Given our patient's epidemiologic risk and presenting features, the most likely infectious etiologies included dengue fever and chikungunya infection, with parvovirus B19 infection less likely. Disease characteristics, clinical features and laboratory data comparing dengue and chikungunya infection are presented in Table 2. Non-infectious causes were deemed unlikely based on the patient's initial fever, rash, and rapid improvement in symptoms.

\section{Table 2: Clinical and laboratory features of chikungunya versus dengue fever}

\begin{tabular}{|l|l|l|}
\hline \multicolumn{1}{|c|}{ Clinical and laboratory features } & \multicolumn{1}{|c|}{ Chikungunya } & \multicolumn{1}{|c|}{ Dengue } \\
\hline Illness Characteristics (19) & $\begin{array}{l}\text { 3-7 days } \\
\text { (range 2-12) }\end{array}$ & $\begin{array}{l}4-7 \text { days } \\
\text { (range 3-14) }\end{array}$ \\
\hline Incubation period & $0.03: 1-0.25: 1$ & $2: 1-10: 1$ \\
\hline $\begin{array}{l}\text { Asymptomatic: } \\
\text { Symptomatic ratio }\end{array}$ & Common & Common \\
\hline Clinical Features (3, 8, 9,17,19,20,21) & Common & Possible \\
\hline Fever & Common & Unlikely \\
\hline Arthralgia & Possible & Common \\
\hline Polyarthritis (without effusion) & $\begin{array}{l}\text { Common, often day } \\
1-4 \text { of illness }\end{array}$ & $\begin{array}{l}\text { Common, often day } \\
3-7 \text { of illness }\end{array}$ \\
\hline Myalgia & Unlikely & Possible \\
\hline Rash &
\end{tabular}




\begin{tabular}{|l|l|l|}
\hline Retro-orbital Pain & Unlikely & Common \\
\hline Chronic joint pains & $\begin{array}{l}\text { Common, can last }>2 \\
\text { years }\end{array}$ & Unlikely \\
\hline Chronic fatigue & $\begin{array}{l}\text { Common, can last }>2 \\
\text { years }\end{array}$ & $\begin{array}{l}\text { Common, up to 3 } \\
\text { months }\end{array}$ \\
\hline Laboratory & Possible & Common \\
\hline Neutropenia & Common & Common \\
\hline Lymphopenia & Possible & Common \\
\hline Thrombocytopenia &
\end{tabular}

The brisk improvement in the patient's symptoms without supportive care is atypical for chikungunya infection. Although likely related to an atypical presentation of disease, the therapeutic effect of chloroquine to mitigate the symptoms of chikungunya infection has been suggested by in vitro studies (15). However, this effect has not been confirmed by randomized controlled trials in humans (16); thus, it is unclear whether his chloroquine antimalarial prophylaxis attenuated his symptoms.

Constipation leading to abdominal pain is also not a classic feature of chikungunya infection. In a comparative study performed in India, 0 of $131(0 \%)$ patients with acute chikungunya infection reported abdominal pain compared with 22 of $104(21 \%)$ patients with acute dengue (17). However, during an outbreak on Reunion Island, $47 \%$ of patients reported gastrointestinal symptoms, although the number with abdominal pain and/or constipation is not reported (8). Given the time course of illness, constipation appears to be associated with this patient's acute chikungunya infection in this case; however, we acknowledge that there may have been two underlying diseases contributing to his symptoms. To explain his constipation and abdominal pain, co-infection with Salmonella spp. enteritis remains a possibility, as this infection is frequently associated with constipation and is endemic to Haiti. Stool cultures in this case were drawn only after administration of antibiotics, which substantially decreases their yield and may have been falsely negative.

\section{Conclusion}

Chikungunya infection is becoming a global concern as countries reporting new transmissions increase. The ability for viral mutation under selective pressure along with growing worldwide travel gives chikungunya significant epidemic potential. Given the varied clinical features at presentation, clinicians need to be vigilant in considering chikungunya infection in patients returning from high-risk countries with fever and polyarthralgia, regardless of other clinical and laboratory features. Although treatment is generally supportive, patient symptoms, including a debilitating arthritis, can persist for years, stressing the importance of patient education in appropriate mosquito bite prevention techniques during travel, including use of DEET- or icaridin-based insect repellants and protective clothing (18).

\section{Conflict of interest}

None

\section{Funding}

None

\section{References}

(1) Deilgat M, Geduld J, Drebot, M. Chikungunya outbreak in the Caribbean 2013-2014. CCDR. 2014 Jan;40(2):7-12. www.phac-aspc.gc.ca/publicat/ccdr-rmtc/14vol40/dr-rm40-02/dr-rm40-02-chik-eng.php

(2) Centers for Disease Control and Prevention. Chikungunya in the Caribbean. www.nc.cdc.gov/travel/notices/watch/chikungunya-caribbean

(3) Thiboutot MM, Kannan S, Kawalekar OU, Shedlock DJ, Khan AS, Sarangan G, et al. Chikungunya: A potentially emerging epidemic? PLoS Negl Trop Dis. 2010;4:e623.

(4) Lumsden WH. An epidemic of virus disease in Southern Province, Tanganyika Territory, in 1952-53. II. General description and epidemiology. Trans R Soc Trop Med Hyg. 1955 Jan;49(1):33-57.

(5) Centers for Disease Control and Prevention. Geographic distribution-Where has chikungunya virus been found? www.cdc.gov/chikungunya/geo/index.html 
(6) Rezza G, Nicoletti L, Angelini R, Romi R, Finarelli AC, Panning M. Infection with chikungunya virus in Italy: An outbreak in a temperate region. Lancet. 2007 Dec 1;370(9602):1840-6.

(7) McCarthy M. First case of locally acquired chikungunya is reported in US. BMJ. 2014 Jul 18;349.

(8) Borgherini G, Poubeau P, Staikowsky F, Lory M, Le Moullec N, Becquart JP, et al. Outbreak of chikungunya on Reunion Island: early clinical and laboratory features in 157 adult patients. Clin Infect Dis. 2007;44:1401-7.

(9) Marimoutou C, Vivier E, Oliver M, Boutin JP, Simon F. Morbidity and impaired quality of life 30 months after chikungunya infection: Comparative cohort of infected and uninfected French military policemen in Reunion Island. Medicine (Baltimore). 2012 Jul;91(4):212-9.

(10) Niedrig M, Zeller H, Schuffenecker I, Drosten C, Emmerich P, Rumer L, et al. International diagnostic accuracy study for the serological detection of chikungunya virus infection. Clin Microbiol Infect. 2009;15:880-4.

(11) Schwartz KL, Giga A, Boggild AK. Chikungunya fever in Canada: Fever and polyarthritis in a returned traveller. CMAJ. 2014 Jul 8;186(10):772-4.

(12) Chang LJ, Dowd KA, Mendoza FH, Saunders JG, Sitar S, Plummer SH. Safety and tolerability of chikungunya virus-like particle vaccine in healthy adults: A phase 1 dose-escalation trial. Lancet. 2014 Dec 6;384(9959):2046-52. http://www.thelancet.com/journals/lancet/article/PIIS0140-6736(14)61185-5/fulltext

(13) Powers AM. Chikungunya virus control: Is a vaccine on the horizon? Lancet. 2014 Dec 6;384(9959):2008-9. http://www.thelancet.com/journals/lancet/article/PIIS0140-6736(14)61290-3/fulltext

(14) Rajapakse S, Rodrigo C, Rajapakse A. Atypical manifestations of chikungunya infection. Trans R Soc Trop Med Hyg. 2010 Feb;104(2):89-96.

(15) Khan M, Santhosh SR, Tiwari M, Lakshmana Rao PV, Parida M. Assessment of in vitro prophylactic and therapeutic efficacy of chloroquine against Chikungunya virus in vero cells. J Med Virol. 2010 May;82(5):817-24.

(16) Chopra A, Saluja M, Venugopalan A. Effectiveness of chloroquine and inflammatory cytokine response in patients with early persistent musculoskeletal pain and arthritis following chikungunya virus infection. Arthritis Rheumatol. 2014 Feb;66(2):319-26.

(17) Taraphdar D, Sarkar A, Mukhopadhyay BB, Chatterjee S. A comparative study of clinical features between monotypic and dual infection cases with Chikungunya virus and dengue virus in West Bengal, India. Am J Trop Med Hyg. 2012 Apr;86(4):720-3.

(18) Schofield S, Plourde P, for the Committee to Advise on Tropical Medicine and Travel (CATMAT). Statement on Personal Protective Measures to Prevent Arthropod Bites. CCDR 2012;38(ACS-3):1-18.

(19) Chen LH, Wilson ME. Dengue and chikungunya in travelers. Curr Opin Infect Dis. 2010 Oct;23(5):438-44.

(20) Queyriaux B, Simon F, Grandadam M, Michel R, Tolou H, Boutin JP. Clinical burden of chikungunya virus infection. Lancet Infect Dis. 2008 Jan;8(1):2-3.

(21) Potts JA, Rothman AL. Clinical and laboratory features that distinguish dengue from other febrile illnesses in endemic populations. Trop Med Int Health. 2008 Nov; 13(11):1328-40. 